\title{
Condition Indicators for Gearbox Condition Monitoring Systems
}

\author{
P. Večeř, M. Kreidl, R. Šmíd
}

Condition monitoring systems for manual transmissions based on vibration diagnostics are widely applied in industry. The systems deal with various condition indicators, most of which are focused on a specific type of gearbox fault. Frequently used condition indicators (CIs) are described in this paper. The ability of a selected condition indicator to describe the degree of gearing wear was tested using vibration signals acquired during durability testing of manual transmission with helical gears.

Keywords: damage detection, condition monitoring, condition indicators, transmissions, vibration.

\section{Introduction}

Condition monitoring systems are very important for researchers in gearbox development. They enable detection of gear cracks during testing, and stop the test before the gear crack progresses. Then the researchers are able to recognize where the crack began and to decide about the reason for the gearbox fault. Consequently, the designers can take appropriate steps in gearbox design to improve gearbox performance.

Condition monitoring systems deal with various types of input data, for instance vibration, acoustic emission, temperature, oil debris analysis etc. Systems based on vibration analysis, acoustic emission and oil debris are the most common and are very well established in industry.

Systems based on acoustic emission have a more obvious application for bearing monitoring than for gearing monitoring. However some applications for gearbox condition monitoring have been introduced. Acoustic emission (AE) is usually defined as transient elastic waves generated from a rapid release of strain energy caused by a deformation or by damage within or on the surface of the material [1]. Successful application of $\mathrm{AE}$ to bearing condition monitoring has been presented in many papers. Roger in [2] describes the application for monitoring of a slowly rotating anti-friction slew bearing mounted in cranes for gas production. Al-Ghamdi and his colleges describe in [3] an experiment where with the use of basic CIs (RMS and max. amplitude) they try to identify the type and size of a bearing defect. They claim that $\mathrm{AE}$ is more sensitive to defect identification than vibration analysis and that the AE burst duration may indicate the size of the bearing defect. Some authors describe the potential of AE to gearbox condition monitoring. Singh [4], Tandom [5] and Sires [6] use simulated gearbox defects and sensor placed on bearings or on a gearbox housing. Toutountzakis [7], Sentoku [8] and Miyakchika [9] present applications to natural defects and they use of a slip ring to transfer the data from a rotating sensor. This type of sensor mounting ensures a direct transfer path for the AE signal. Tan [10] deals with the sources of AE during meshing of spur gears. He offers three possible sources of $\mathrm{AE}$ during the mesh: tooth resonance, secondary pressure peak in lubricated gears and asperity contacts. He considers the asperity contact to be the most important source of AE.

Oil debris analysis is a very reliable method for detecting gearing damage in the early stages and allows estimation of the wear level. During gearbox operations the contacting surfaces of gearwheels and bearings are gradually abraded. Small pieces of material break down from the contact surfaces. These small pieces of material are carried away by oil lubricating the gearwheels and bearing. By detecting the number and size of particles in the oil we can identify gear-pitting damage in an early stage, which is unidentifiable by vibration analysis.

Oil debris sensors are usually based on a magnetic or an optical principle. Magnetic sensors measure the change in magnetic field caused by metal particles in a monitored sample of oil.

The oil debris monitoring system can be on-line or off-line. Oil debris monitoring systems are usually more reliable than vibration based systems for early pitting failure detection [11]. A disadvantage of oil debris analysis is that it does not localize the failure in complicated gearboxes. The oil used in the oil debris monitoring system should not dissolve the metal particles and spread a metal film on to the gearbox housing.

Condition monitoring systems based on vibration analysis can monitor all parts of gearboxes, for example gearing, bearings and shafts.

A typical condition monitoring system based on vibration monitoring is depicted in Fig. 1.

For a better signal to noise ratio a raw vibration signal is filtered and pre-amplified. Consequently the signal is processed in two different ways. The overall vibration level is monitored in an analog RMS detector. If the vibrations exceed the selected level the system stops the gearbox testing before the gearbox is destroyed. To set the system more sensitive to incipient gearbox failures, the analog signal is digitized. The time domain signal is synchronously averaged and consequently filtered to focus only on the important part of the vibration signal. Then some condition indicators (CIs) are computed. Finally the computed CIs are compared in the decision making unit. If any of the indicators exceed the limit the alarm signal is generated. Sometimes single condition indicators are not used and complete Fourier spectra are compared.

Since 1997, when Steward introduced FM0 and FM4 condition indicators and some other CIs [12], research in gearing condition indicators has progressed. Zakrejsek [13] introduced NA4 which is sensitive to damage growth. NA4 was further improved in trending by Decker [14] and also by Demsey [15] to decrease NA4 dependency on torque changes. 


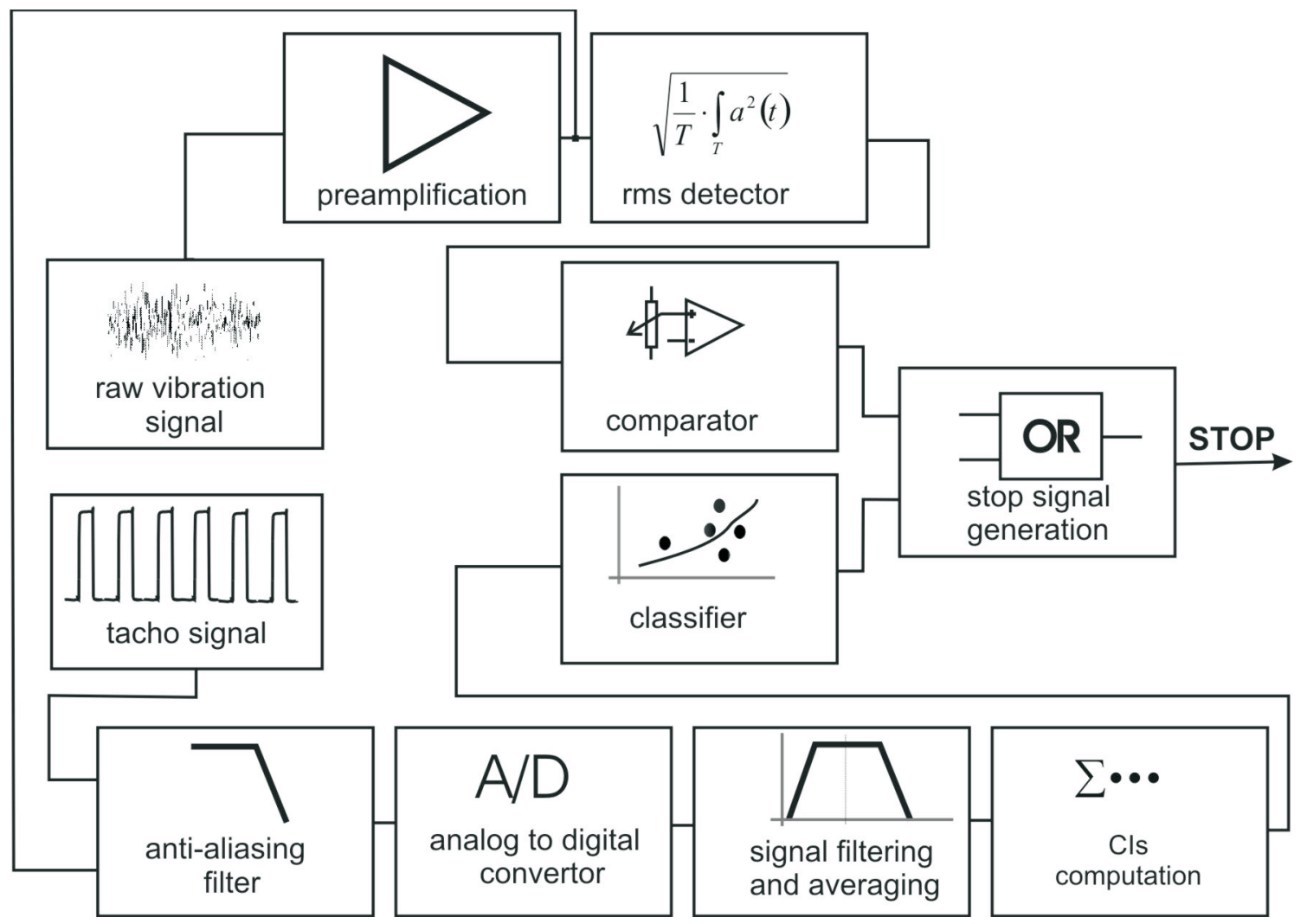

Fig. 1: Condition monitoring system based on vibration analysis

Zakrejsek [13] presented the application in gearbox diagnostics of CI M6A and M8A, originally developed by Martin [17] for surface damage detection.

\section{Description of gearbox condition indicators}

Traditional CIs deal with data distribution. The main differences between these CIs are in the signal from which the computations are made.

Generally three type of signal are used: a raw, a residual and a difference signal. A residual signal is defined as a synchronous averaged signal without the gear mesh frequency, its harmonics, driveshaft frequency and its second harmonics. If the first order sidebands about the gear mesh frequencies are filtered out, a differential signal is created. However these definitions are not strict. Some authors leave the second harmonics of the driveshaft frequency in the residual signal or remove the second order sideband from the difference signal.

\subsection{Root mean square value}

The Root Mean Square value (RMS) for the velocity vibration signal is defined in Equation 1. In comparison with the definition of kinetic energy $E_{K}$ (Eq. 2) it is obvious that the RMS value computed from the velocity of the vibration signal describes the energy content of the signal.

$$
v_{r m s}=\sqrt{\frac{1}{T} \int_{T} v^{2}(t) \mathrm{d} t},
$$

where $v_{r m s}$ is the root mean square value of the velocity of the vibration signal,

$T$ is integration time,

$v \quad$ is the velocity of the moving object.

$$
E_{K}=\frac{1}{2} m v^{2}(t),
$$

where $E_{K} \quad$ is the kinetic energy,

$m \quad$ is the weight of the moving object.

Nowadays, however, digital signals are more used than analog signals. The RMS definition for a discrete signal is given in Equation 3.

$$
s_{r m s}=\sqrt{\frac{1}{N} \sum_{i=1}^{N}\left(s_{i}^{2}\right)},
$$

where $s_{r m s}$ is the root mean square value of dataset $s$,

$s_{i} \quad$ is the $i$-th member of dataset $s$,

$N \quad$ is the number of points in dataset $s$.

From the definition of RMS it is obvious that the RMS value does not increase with the isolated peaks in the signal, consequently this parameter is not sensitive to incipient tooth failure. Its value increases as tooth failure progresses. Generally the RMS value of the vibration signal is a very good descriptor of the overall condition of the tested gearboxes. This parameter is sensitive to gearbox load and speed changes. The main usage of this parameter is to monitor the overall vibration level. Then the test can be stopped when the vibration energy reaches the critical value and the gearbox destruction can be treated. Typical time history of the RMS 
value for the overall vibration signal during a gearbox durability test is depicted in Fig. 2.

As the gearbox consequently wears out, the vibration level will increase. When pitting damage occurs, the vibration level will increase. As pitting damage progresses, the overall vibration level increases rapidly.

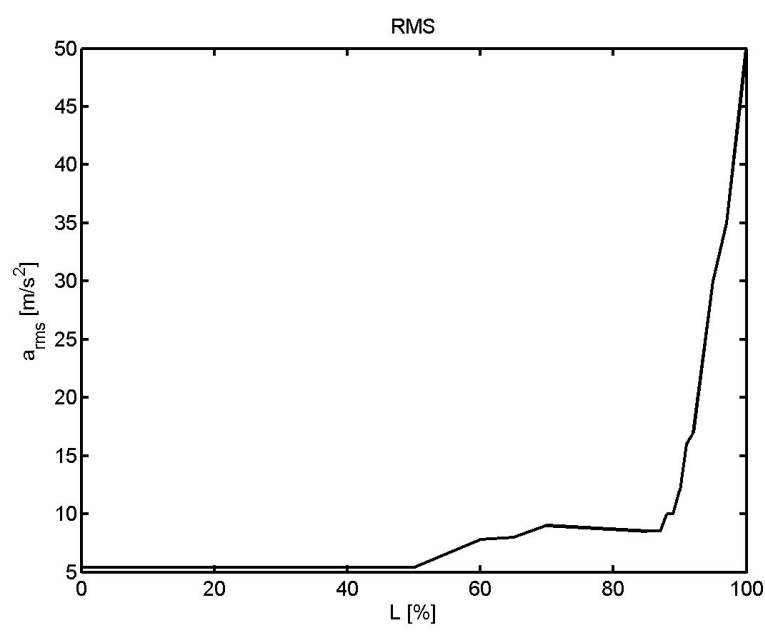

Fig. 2: Time history of the vibration signal during a durability test of the gearing ( $L$ is the duration of the test normalized by the total test duration)

\subsection{Delta RMS}

This parameter is the difference between two consequent RMS values. This parameter focuses on the trend of the vibration and is sensitive to vibration signal changes. Theoretically it allows selection of an alarm level which is not sensitive to load. However the parameter is sensitive to load change. The theory behind this parameter states that if gear damage occurs the vibration level will be increased more rapidly than in a normal case without gear damage.

\subsection{Peak value}

This value is the maximum value of the signal in a selected time interval. This parameter is usually not used alone.

\subsection{Crest factor}

This parameter indicates the damage in an early stage. It is defined as the peak value of the signal divided by the RMS value of the signal.

$$
C F=\frac{s_{\text {peak }- \text { peak }}}{s_{r m s}},
$$

where $C F \quad$ is the crest factor,

$s_{\text {peak-peak }}$ is the peak to peak value of the signal,

$s_{r m s} \quad$ is the root mean square value of the vibration signal.

When only one tooth is damaged, there is no change in the RMS value of the vibration signal during one rotation of the drive shaft where the damaged gear is located, while the peak value increases. Therefore the crest factor increases its value. As the damage progresses the root mean square value of the vibration signal increases its value and the crest factor decreases. This parameter enables very tiny surface damages to be discovered, as experiments show. The crest factor is often used in gearbox quality monitoring devices.

\subsection{Energy operator}

The energy operator $(E O)$ is computed as the normalized kurtosis from the signal where each point is computed as the difference of two squared neighborhood points of the original signal [18].

$$
E O=\frac{N^{2} \sum_{i=1}^{N}\left(\Delta x_{i}-\Delta \bar{x}\right)^{4}}{\left(\sum_{i=1}^{N}\left(\Delta x_{i}-\Delta \bar{x}\right)^{2}\right)^{2}}
$$

where $E O \quad$ is the energy operator,

$\Delta \bar{x} \quad$ is the mean value of signal $\Delta x$, $\Delta x_{i}=s_{i+1}^{2}-s_{i}^{2}$,

$N \quad$ is the number of the point in dataset $x$.

\subsection{Kurtosis}

The shape of the amplitude distribution is often used as a data descriptor. Kurtosis describes how peaked or flat the distribution is. If a vibration signal contains sharp peaks with a higher value, then its distribution function will be sharper. We can assume that these types of signals will be produced by a damaged gearbox. Therefore the kurtosis value will be higher for a damaged gearbox than for a gearbox in good condition.

A mathematical definition of kurtosis is given by Eq. 6 .

$$
\text { Kurt }=\frac{N \cdot \sum_{i=1}^{N}\left(s_{i}-\bar{s}\right)^{4}}{\left(\sum_{i=1}^{N}\left(s_{i}-\bar{s}\right)^{2}\right)^{2}},
$$

where Kurt is kurtosis,

$N \quad$ is the number of points in the time history of signal $s$,

$s_{i} \quad$ is the $i$-th point in the time history of signal $s$.

Thus kurtosis is the fourth centralized moment of the signal, normalized by the square of the variance.

\subsection{Energy ratio}

The energy ratio $(E R)$ is defined as the ratio between the energy of the difference signal and the energy of the regular meshing component [19].

$$
E R=\frac{\sigma(d)}{\sigma(r)},
$$

where $E R \quad$ is energy ratio,

$\sigma(d) \quad$ is the standard deviation of the difference signal,

$\sigma(r) \quad$ is the standard deviation of the regular signal.

Not all authors use the same definition for these signals. The regular meshing components are usually defined as the mesh frequency and its harmonics. Consequently the differ- 
ence signal is defined as the remainder of the vibration signal after the regular meshing components are removed.

The basic idea of this indicator is that the energy is transferred from the regular meshing component to the rest of the signal as the wear progresses. This parameter is a very good indicator for heavy wear, where more than one tooth on the gearing is damaged.

\subsection{Sideband level factor}

The sideband level factor is defined as the sum of the first order sideband about the fundamental gear mesh frequency divided by the standard deviation of the time signal average [20]. This parameter is based on the idea that tooth damage will produce amplitude modulation of the vibration signal. For a gearbox in good condition this factor is near zero.

\subsection{Sideband index}

The sideband index is defined as the average amplitude of the sidebands of the fundamental gear mesh frequency [21].

\subsection{Zero-order figure of merit}

The zero-order figure of merit $(F M 0)$ parameter is defined as the quotient of the peak-to-peak value of the signal divided into the energy of the mesh frequency and its harmonics [21]. It is obvious that this parameter is similar to the crest factor. FM0 compares the peak value of the synchronous averaged signal to the energy of the regular signal, and the crest factor compares the peak value of the synchronous averaged signal to the energy of the synchronous averaged signal. Therefore FMO is more focused on the damage to the tested gearing.

$$
F M 0=\frac{S_{\text {peak-peak }}}{\sum_{i=1}^{N} A(i)}
$$

where FMO is the zero-order figure of merit,

$S_{\text {peak-peak }}$ is the peak to peak value of the vibration signal in the time domain,

$A(i) \quad$ is the amplitude of the $i$-th mesh frequency harmonics.

Let us assume that one tooth on the gear mesh gear is slightly damaged. Then the gearing produces a vibration signal with a significantly increased peak to peak value. However, the sum of the root mean square values is approximately the same.

\subsection{FM4 parameter}

The FM4 parameter [18] is a simple measure if the amplitude distribution of the difference signal is peaked or flat. The parameter assumes that a gearbox in good condition has a difference signal with a Gaussian amplitude distribution, whereas a gearbox with defective teeth produces a difference signal with a major peak or a series of major peaks resulting in a less peaked amplitude distribution. If more then one tooth is defective, the data distribution becomes flat and the kurtosis value decreases.

$$
F M 4=\frac{N \cdot \sum_{i=1}^{N}\left(d_{i}-\bar{d}\right)^{4}}{\left(\sum_{i=1}^{N}\left(d_{i}-\bar{d}\right)^{2}\right)^{2}}
$$

where $d_{i} \quad$ is the $i$-th point of the differential signal in the time record,

$N \quad$ is the total number of points in the time record.

\subsection{NA4 parameter}

The NA4 [18] parameter was developed to improve the behavior of the FM4 parameter when more than one tooth is damaged. The first difference between NA4 and FM4 is that $N A 4$ uses a residual signal to compute kurtosis. The second difference is that we use an average value of variance. Thus if the gear damage spreads from one tooth to another tooth the value of the average variance increases slowly and allows the $N A 4$ parameter to grow. The second reason why the NA4 parameter increases its value is that the residual signal contains the first order sidebands, which increase when tooth damage occurs. The NA4 parameter is defined by Eq. 10 .

$$
N A 4=\frac{N \cdot \sum_{i=1}^{N}\left(r_{i}-\bar{r}\right)^{4}}{\left(\frac{1}{M} \cdot \sum_{j=1}^{M}\left(\sum_{i=1}^{N}\left(r_{i j}-\bar{r}_{j}\right)^{2}\right)\right)^{2}},
$$

where $r_{i} \quad$ is the $i$-th point in the time record of the residual signal,

$$
\begin{aligned}
& r_{i j} \quad \begin{array}{l}
\text { is the } i \text {-th point in the } j \text {-th time record of the } \\
\text { residual signal, }
\end{array} \\
& j \quad \text { is the current time record, } \\
& i \quad \text { is the data point number per reading, } \\
& M \quad \text { is the current time record in the run } \\
& \text { ensemble, } \\
& N \quad \text { is the number of points in the time record. }
\end{aligned}
$$

When gear damage progresses the averaged variance value increases rapidly, which results in a decrease of the $N A 4$ parameter. To overcome this problem the $N A 4^{*}$ parameter was introduced.

The fourth centralized moment of the residual signal is normalized with the average variance for a gearbox in good condition. This allows the $N A 4^{*}$ parameter to grow as the damage progresses.

$$
N A 4^{*}=\frac{N \cdot \sum_{i=1}^{N}\left(r_{i}-\bar{r}\right)^{4}}{\left(\operatorname{var}\left(r_{O K}\right)\right)^{2}},
$$

where $\operatorname{var}\left(r_{O K}\right)$ is the variance for a gearbox in good condition.

The value of the signal for a gearbox in good condition is usually assumed from the variance for a well-functioning gearbox. 


\section{$2.13 \mathrm{NB} 4$ parameter}

The NB4 [22] parameter is designed from the NA4 parameter. NA4 is computed from the residual signal whilst NB4 is computed from the envelope signal. The computation procedure follows. A raw vibration signal is bandpass filtered about the gear meshing frequency. Each recommendation for the bandwidth is different. Some authors suggest using a band pass filter with the bandwidth giving the maximum amount of the sidebands, whilst others use a filter with the bandwidth limited by the first harmonic different from the gear mesh frequency. After the unwanted part of signal has been filtered out, the Hilbert transform (Eq.12) is used to create an analytic signal $\hat{a}$.

$$
\mathcal{H}[a(t)]=\widetilde{a}(t)=\frac{1}{\pi} \int_{-\infty}^{\infty} a(\tau) \cdot \frac{1}{t-\tau} d \tau
$$

Where $\widetilde{a}(t) \quad$ is the Hilbert transform,

$a(\tau) \quad$ is the input real analog signal.

From the analytic signal the envelope is simply calculated according to Eq. 13. The result is the input for the NA4 parameter.
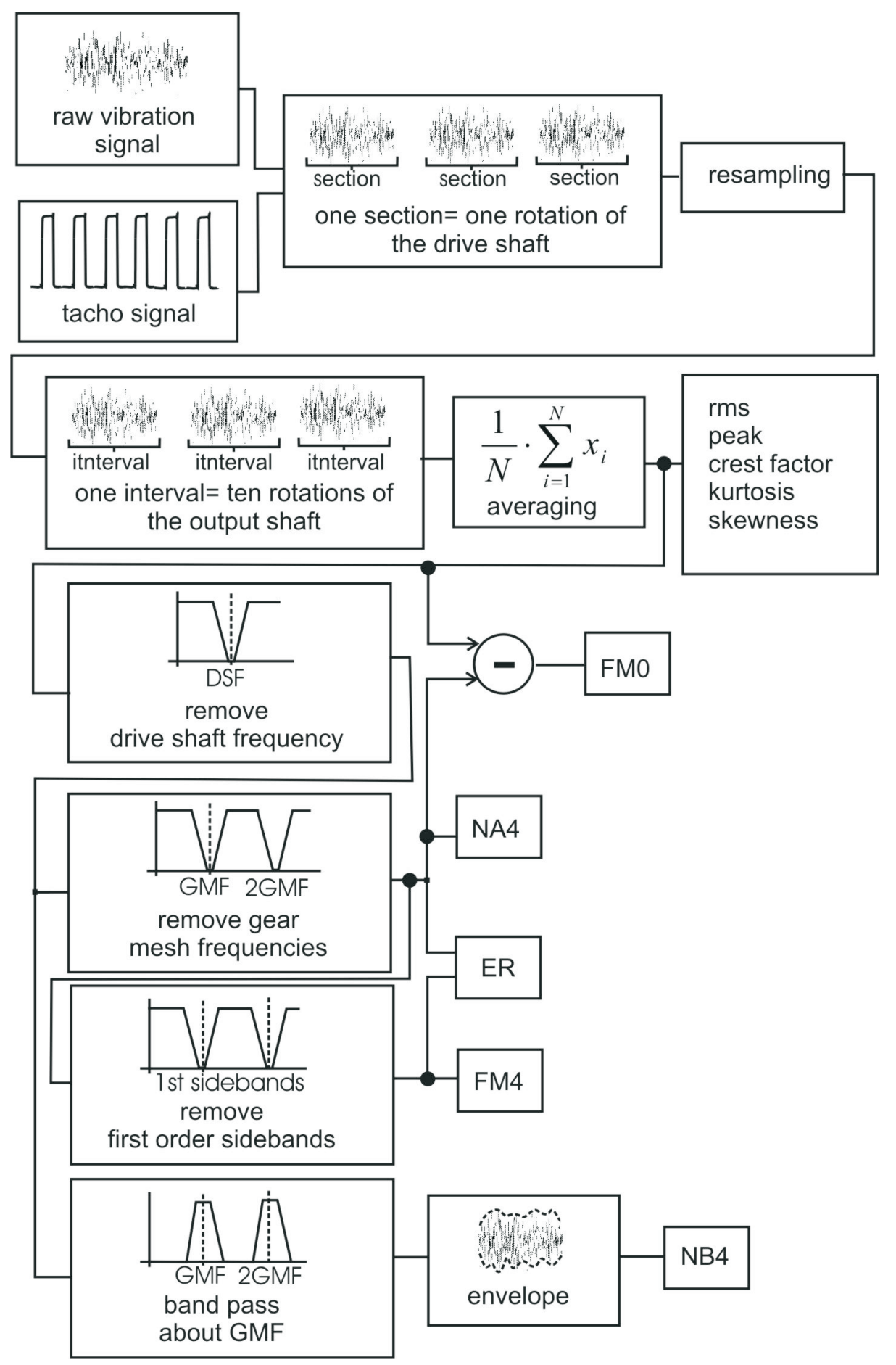

Fig. 3: Signal processing during the test 


$$
|\widehat{a}(t)|=\sqrt{a^{2}(t)+\widetilde{a}^{2}(t)}
$$

where $|\widehat{a}(t)|$ is the envelope of the analytic signal,

$a(t) \quad$ is an input analog signal,

$\widetilde{a}(t)$ is the Hilbert transform of the input signal.

\section{Experiments with selected CIs}

Selected CIs were used to monitor the condition of a gearbox during its durability test. The idea behind the test is to assess the CI indicator which best follows the condition of the gearbox. The CIs were computed for the same input conditions, because of the dependency on torque and rotational speed.

\subsection{Data acquisition}

The vibrations of the gearbox housing were measured during a simulated test drive on the test bench. One piezoelectric accelerometer was used for vibration signal acquisition. The transducer was placed near the differential gearing. The data from the accelerometer was recorded directly onto the PC hard disk for off-line analysis by the B\&K multi-analyzer system type 3560 . The B\&K MM0024 photoelectric tachometer probe captured the rotational speed of the drive shaft during the test procedure.

\subsection{Data processing in the digital domain}

Because of slight speed changes, the raw vibration signal is re-sampled to obtain the same number of samples in each basic time interval. The basic interval matches one rotation of
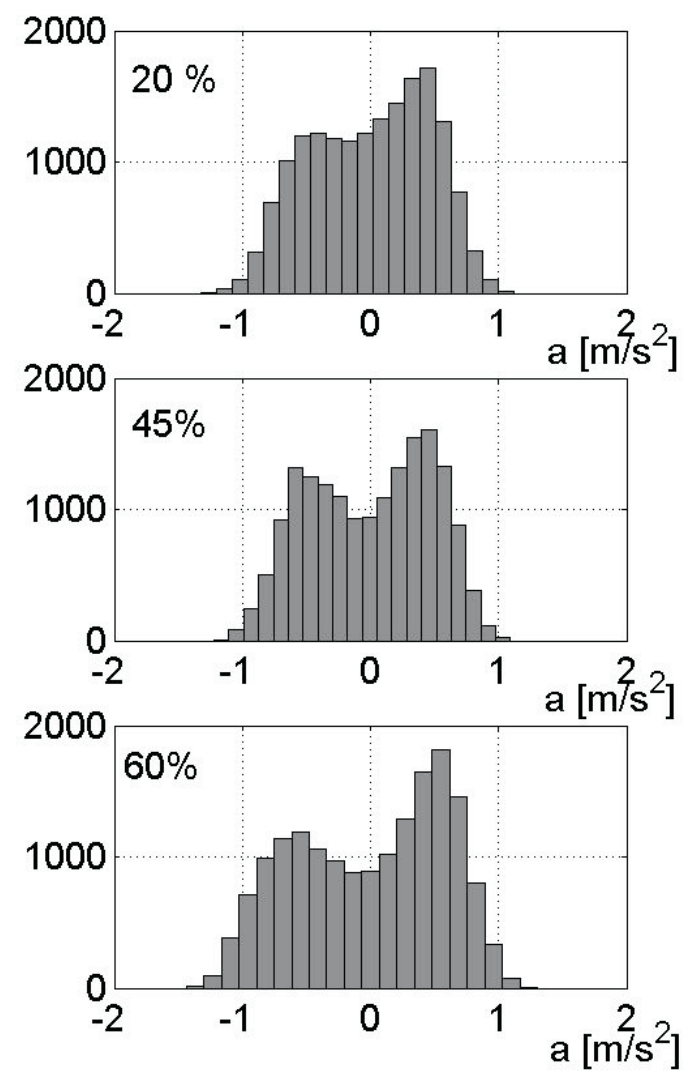

the drive shaft. Then the re-sampled signal is divided into sections. The section lengths correspond to 10 rotations of the output shaft. After that the sections are averaged to decrease the noise. Then the signal is filtered to obtain the residual, difference and regular signal. Consequently the CIs were computed. Mean values of the CIs are used as representative for each CI for a selected length of the input interval. The data processing is depicted in Fig. 3.

\subsection{Experimental results}

The main idea of most CIs is as follows. The amplitude distribution of the vibration signal without gear mesh frequencies differs more from Gaussian distribution as the gearbox wears.

The changes in amplitude distribution during the test are depicted in figure 4 . These changes are numerically expressed by the CIs in Figs. 5 and 6. The condition of the tested gearbox is very well represented by the RMS value of the vibration signal. The trend of the RMS, peak and crest factor value is depicted in Fig. 5. The energy ratio has a steady trend except at the start of the test. The skewness and kurtosis do not reflect any explicit trend. The RMS slightly increases during the test. The peak value has a similar trend as the RMS value. The trend of the crest factor depends on the trends of the RMS and peak values.

FM0 follows the trend of the crest factor. The trend of FM4, NA4 and NB4 are similar, and reflect the very fine wear of the teeth. At about $60 \%$ of the test FM4, NA4 and NB4 rapidly increase in value, but the gearing does not show abnormal wear.
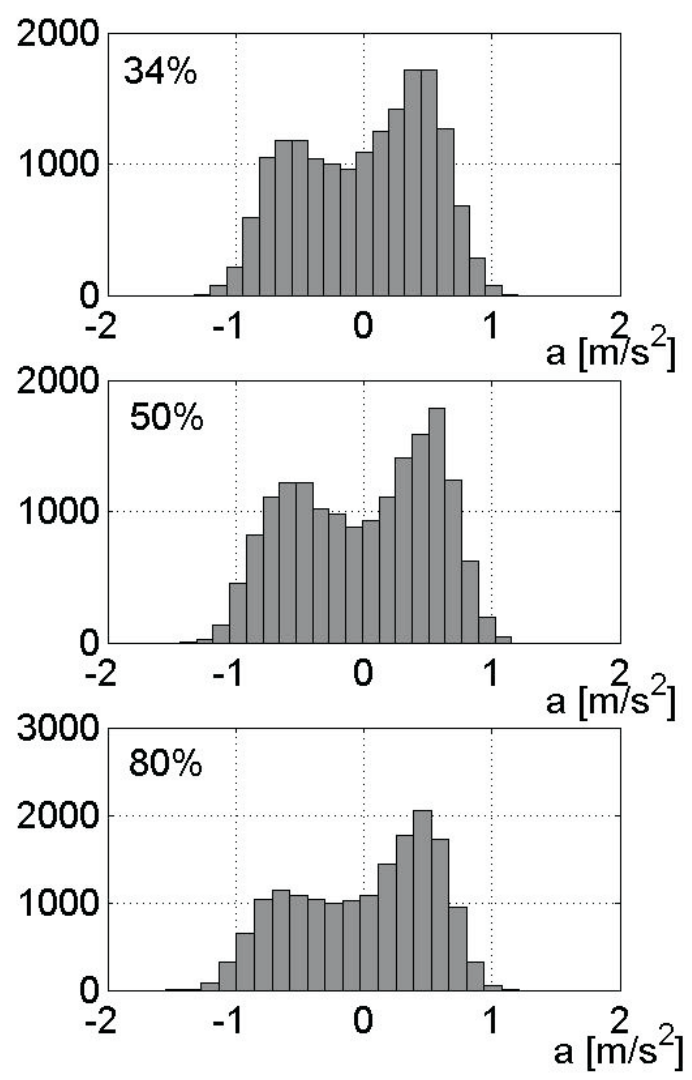

Fig. 4: Histograms of asynchronous averaged signal during the test 

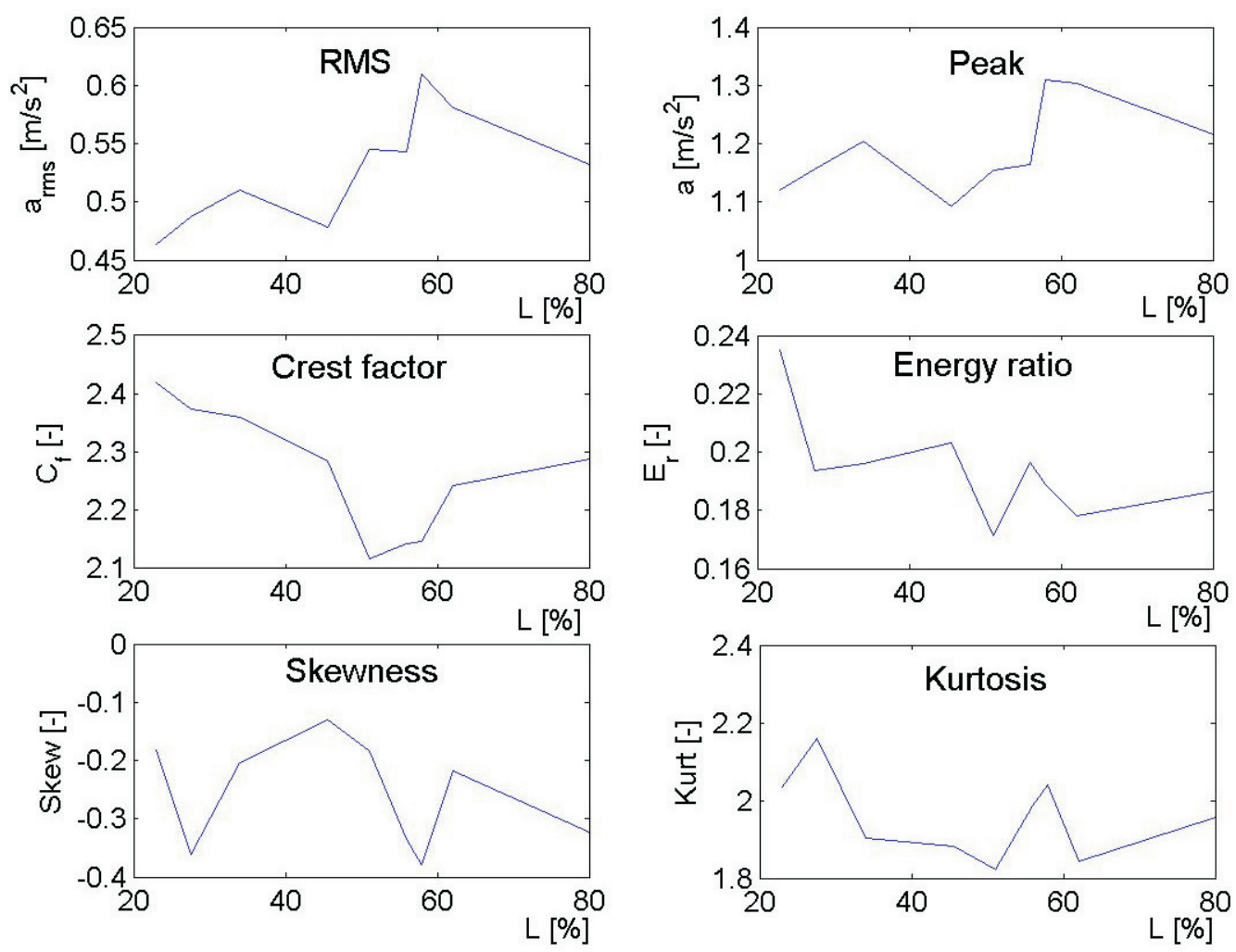

Fig. 5: Trend of RMS, Peak, Crest factor, Energy ratio, Skewness and Kurtosis (L is the actual duration of the test normalized by the total test duration)
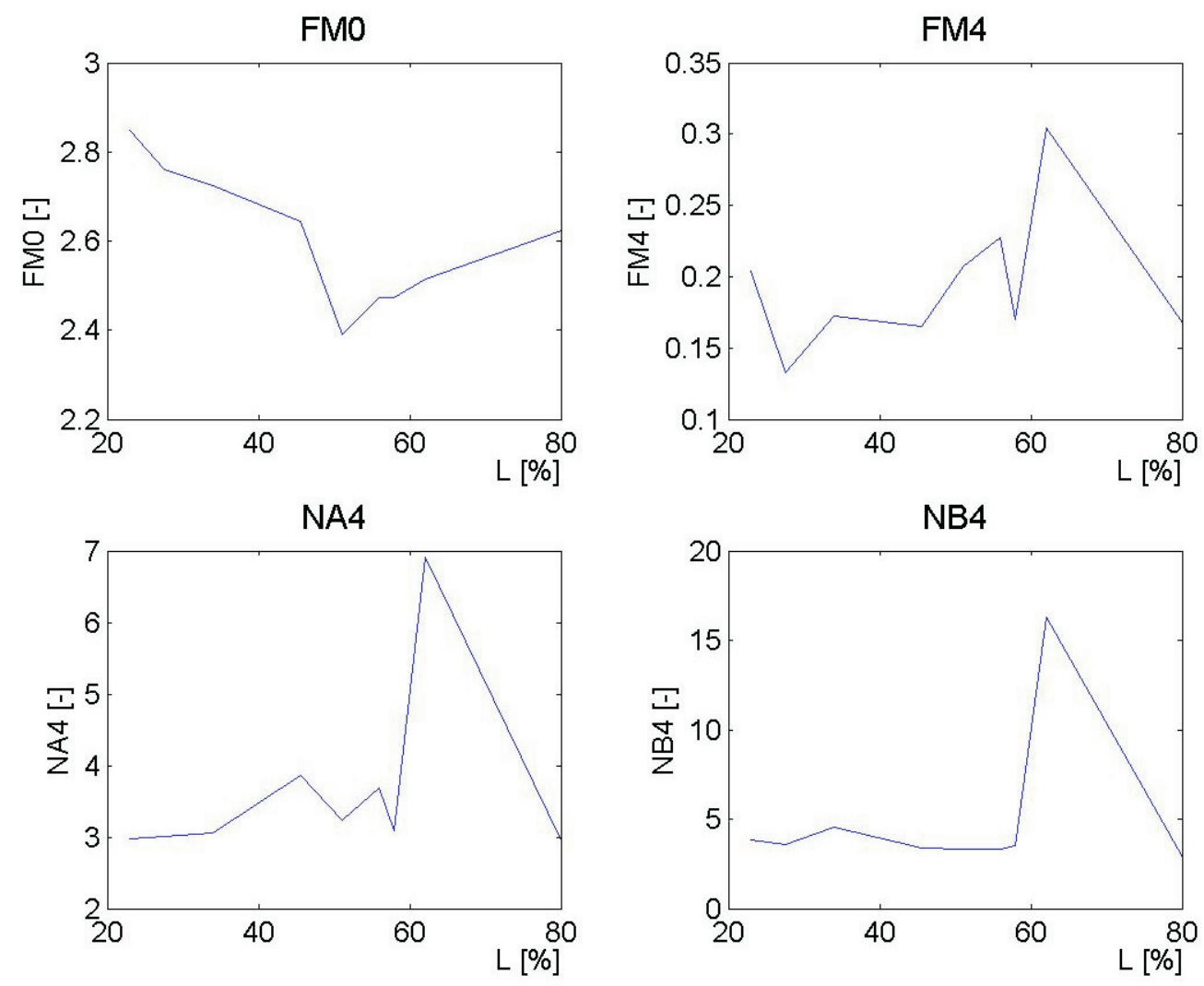

Fig. 6: Trend of the $F M 0, F M 4, N A 4$ and $N B 4$ parameter 


\section{Conclusion}

Condition indicators are based on detecting of differences between the amplitude distribution of a vibration signal without filtered gear mesh frequencies and the distribution of the signal for a gearbox in good condition. The rest of the vibration signal without the gear mesh frequency, its harmonics and shaft frequency has its amplitude distribution near to a normal distribution for a gearbox in good condition. Therefore many CIs give a zero value for a gearbox in good condition. CIs differ each from other in the signal that they use and how they use the time history of the vibration signal. Some of them are improved for torque independency.

The experimental result indicates that condition indicators describing the overall vibration level track the condition of the tested gearbox condition (rms, peak) very well. Typical gearbox monitoring systems are therefore based on order analysis and condition indicators describing the overall vibration value (rms, crest factor etc.) Condition indicators provide information that something has happened and order analysis provides information about what has happened.

The condition indicator that indicates the gearbox fault at its beginning does not track the condition of the gearbox well during the test, e.g., the crest factor. The author ascribes this to the fact that the condition indicator was not computed continuously during the test. It was computed only from the selected datasets of the vibration signal acquired during the test.

The experimental results indicate that the monitoring of fine wear of gearing places big demands on precise data acquisition. The part of the vibration signal created by gearing wear can be masked by noise. In these cases, an additional method such as oil debris should be used for precise condition monitoring.

\section{Acknowledgments}

The research was supported by the research program No. MSM6840770015 "Research of Methods and Systems for Measurement of Physical Quantities and Measured Data Processing" of the CTU in Prague sponsored by the Ministry of Education, Youth and Sports of the Czech Republic.

\section{References}

[1] Mathews, J. R.: Acoustic Emission. Gordon and Breach Science Publishers Inc., New York 1983.

[2] Roger, L. M.: "The Application of Vibration Analysis and Acoustic Emission Source Location to On-Line Condition Monitoring of Anti-Friction Bearings.” Tribology International, 1979, p. 51-59.

[3] Al-Ghamdi, A. M., Zhechkov, D., Mba, D.: "The Use of Acoustic Emission for Bearing Defect Identification and Estimation of Defect Size." DGZfP-Proceedings BB 90, 2004.

[4] Singh, A., Houser, D. R., Vijayakar, S.: "Detection of Gear Pitting." Power Transmission and Gearing Conference, 1996, ASME. DE-Vol. 88, p. 673-678.
[5] Tandom, N., Mata, S.: "Detection of Defects in Gears by Acoustic Emission Measurements." Journal of Acoustic Emission, Vol. 17 (1999), Issue 1-2, p. 23-27.

[6] Siores, E., Negro, A. A.: "Condition Monitoring of a Gear Box Using Acoustic Emission Testing, Material Evalution.”, 1997, p. 183-187.

[7] Toutountzakis, T., Mba, D.: "Observation of Acoustic Emission Activity During Gear Defect Diagnostis.” NDT and E International. Vol. 26 (2003), p. 471-477.

[8] Sentoku, H.: "AE in Tooth Surface Failure Process of Spur Gears.” Journal of Acoustic Emission, Vol. 16 (1998), Issue 1-4, S19-S24.

[9] Miyachika, K., Oda, S., Koide, T.: “Acoustic Emission of Bending Fatigue Process of Spur Gear Teeth.” Journal of Acoustic Emission, Vol. 13 (1995), Issue 1-2, S47-S53.

[10] Tan, C. K., Mba, D.: "The Source of Acoustic Emission During Meshing of Spur Gears.” EWGAE 2004.

[11] Dempsey, P. J., Afjeh, A. A.: "Integrating Oil Debris and Vibration Gear Damage Detection Technologies Using Fuzzy Logic." International $58^{\text {th }}$ Annual Forum and Technology Display, Quebec (Canada) June 11-13, 2002.

[12] Stewart, R. M.: "Some Useful Data Analysis Techniques for Gearbox Diagnostics" Report MHM/R/10/77, Machine Health Monitoring Group, Institute of Sound and Vibration Research, University of Southampton, July 1977.

[13] Zakrajsek, J. J., Townsend, D. P., Decker, H. J.: “An Analysis of Gear Fault Detection Methods as Applied to Pitting Fatigue Failure Data.” NASA TM-105950, presented at $47^{\text {th }}$ Meeting of the Society for Machinery Failure Prevention Technology, April 1993.

[14] Decker, H. J., Handschuh, R. F., Zakrajsek, J. J.: “An Enhancement to the NA4 Gear Vibration Diagnostic Parameter, $18^{\text {th }}$ Annual Meeting Vibration Institute, Hershey, PA, June 20-23, 1994.

[15] Dempsey, P. J., Zakrajsek, J. J.: "Minimizing Load Effects on NA4 Gear Vibration Diagnostic Parameter." NASA TM-2001-210671, Glenn Research Center, Cleveland, OH, Feb. 2001.

[16] Martin, H. R.: "Statistical Moment Analysis as a Means of Surface Damage Detection." Proc. of $7^{\text {th }}$ International Modal Analysis Conference, Society for Experimental Mechanics, Schenectady, N.Y., 1989.

[17] Mosher, M., Pryor A. H., Huff, E. M.: "Evaluation of Standard Gear Metrics in Helicopter Flight Operation." $56^{\text {th }}$ Mechanical Failure Prevention Technology Conference, Virginia Beach, VA, April 15-19, 2000.

[18] Decker, H. J.: "Crack Detection for Aerospace Quality Spur Gears." $58^{\text {th }}$ International Annual Forum and Technology Display sponsored by the American Helicopter Society, Montreal, Quebec, Canada, June 11-13, 2002.

[19] Keller, J. A., Grabill, P.: "Vibration Monitoring of UH-60A Main Transmission Planetary Carrrier Fault." American Helicopter Society $59^{\text {th }}$ Annual Forum, Phonix, Arizona, May 6-8, 2003. 
[20] Szczepanik A.: "Time Synchronous Averaging of Ball Mill Vibration." Mechanical Systems and Signal Processing, Vol. 3 (1989), No. 1, p. 99-107.

[21] Stewart, R. M.: "Some Useful Data Analysis Techniques for Gearbox Diagnostics.” Report MHM/R/10/77, Machine Health Monitoring Group, Institute of Sound and Vibration Research, University of Southampton, July 1977.

[22] Lebold, M., McClintic, K., Cambell, R., Byington, C., Maynard, K.: "Review of Vibration Analysis Methods for Gearbox Diagnostics and Prognostics." Proceedings of the $54^{\text {th }}$ Meeting of the Society for Machinery Failure Prevention Technology, Virginia Beach,VA, May 1-4, 2000, p. 623-624.

[23] Dempsey, P. J.: “A Comparison of Vibration and Oil Debris Gear Damage Detection Methods Applied to Pitting Damage." $13^{\text {th }}$ International Congress on Condition Monitoring and Diagnostic Engineering Management sponsored by the Society for Machinery Failure Prevention Technology, Houston, Texas, December 3-8, 2000.
[24] Dempsey, P. J., Zakrajsek, J. J.: "Minimizing Load Effects on NA4 Gear Vibration Diagnostic Parameter." $55^{\text {th }}$ Meeting sponsored by the Society for Machinery Failure Prevention Technology, Virginia Beach, Virginia, April 2-5, 2001.

Ing. Petr Večeř

e-mail: vecerp@fel.cvut.cz

Doc. Ing. Marcel Kreidl, CSc.

phone: +420224352117

e-mail: kreidl@fel.cvut.cz

Doc. Ing. Radislav Šmíd, Ph.D.

phone: +420224352131

e-mail:smid@fel.cvut.cz

Department of Measurements

Czech Technical University in Prague

Faculty of Electrical Engineering

Technická 2

16627 Prague 6, Czech Republic 